\title{
Almost perfect powers in arithmetic progression
}

\author{
by \\ N. Saradha and T. N. Shorey (Mumbai)
}

1. Introduction. Let $b, d, \ell, n$ and $y$ be positive integers such that $\operatorname{gcd}(n, d)=1$ and $\ell>2$ is a prime number. Let $k \geq 2, t \geq 2$ and $r \in\{0,1\}$ be integers satisfying $t=k-r$. Thus $k \geq 2$ if $r=0$ and $k \geq 3$ if $r=1$. Let $d_{1}<\ldots<d_{t}$ be integers in the interval $[0, k)$. We write

$$
\Delta=\Delta\left(n, d, k, r, d_{1}, \ldots, d_{t}\right)=\left(n+d_{1} d\right) \ldots\left(n+d_{t} d\right) .
$$

For an integer $\nu>1$, we denote by $P(\nu)$ the greatest prime factor of $\nu$ and we put $P(1)=1$. For $P(b) \leq k$, we consider the equation

$$
\left(n+d_{1} d\right) \ldots\left(n+d_{t} d\right)=b y^{\ell}
$$

in integers $b, d, k, \ell, n, y, d_{1}, \ldots, d_{t}$. If $r=0$, then $d_{i}=i$ for $0 \leq i<k$ and the left hand side of $(1.1)$ is $n(n+d) \ldots(n+(k-1) d)$. If $r=1$, the left hand side of (1.1) is obtained by omitting a term $n+i d$ for some $i$ with $0 \leq i<k$ from $\{n, n+d, \ldots, n+(k-1) d\}$. When considering equation (1.1), it is natural to suppose that

$$
P(\Delta)>k \quad \text { if } d=1
$$

and

$$
P(\Delta)>k \quad \text { if } d>1
$$

the latter inequality holds, by Theorem 4 , unless

$$
r=1, \quad k=3, \quad d>1
$$

or

$$
(n, d, k)=(2,7,3) \quad \text { if } r=0
$$

and

$$
\begin{aligned}
\left(n, d, k, d_{1}, \ldots, d_{t}\right) \in\{ & (1,5,4,0,1,3),(2,7,4,0,1,2),(3,5,4,0,1,3), \\
& (1,2,5,0,1,2,4),(2,7,5,0,1,2,4), \\
& (4,7,5,0,2,3,4),(4,23,5,0,1,2,4)\} \quad \text { if } r=1 .
\end{aligned}
$$

2000 Mathematics Subject Classification: Primary 11D61. 
The above tuples $(n, d, k)$ and $\left(n, d, k, d_{1}, \ldots, d_{t}\right)$ are excluded throughout the paper without reference. We have no contribution on equation (1.1) with $r=1, k=3, d>1$ and therefore we always suppose that $k \geq 4$ if $r=1$ and $d>1$. Thus $P(\Delta)>k$ if $d>1$. Theorem 4 is equivalent to the statement that $\Delta$ with $r=0, d>1$ and $k \geq 4$ is divisible by at least two distinct primes if (3.2) holds (see Theorem $4^{\prime}$ ). This sharpens earlier results of Sylvester [26], Langevin [8] and Shorey and Tijdeman [23].

First, we consider equation (1.1) with $r=0$ and $d=1$. Erdős [3] and Rigge [12], independently, proved that equation (1.1) with $r=0$ and $b=d=1$ implies that $k$ is bounded by a number $C$ depending only on $\ell$. Further, Erdős and Siegel (unpublished) showed that $C$ can be replaced by an absolute constant. The proofs of the preceding results were not elementary and they depend on a deep method of Thue for diophantine equations. An elementary proof of the result of Erdős and Siegel was given by Erdős [4] and this elementary method led Erdős and Selfridge [5] to establish a striking theorem in the theory of exponential diophantine equations that (1.1) with $r=0, d=1, P(b)<k$ and (1.2) has no solution. The method of Erdös and Selfridge is still elementary and it depends on graph theory. The assumption $P(b)<k$ has been relaxed to $P(b) \leq k$ for $k \geq 4$ by Saradha [15] and for $k=2,3$ by Györy [6]. Saradha's proof depends on the method of Erdös and Selfridge whereas Győry derived his results from the deep theorems of Ribet [11] and Darmon and Merel [2] on generalised Fermat equations.

All the constants appearing in this paper from now onward are effectively computable. We write $C_{1}, C_{2}, \ldots$ for effectively computable absolute positive constants. It follows from a result of Shorey [17] that equation (1.1) with $r=d=1$ and (1.2) implies that $k \leq C_{1}$. Further Saradha [15] proved that $C_{1}$ can be taken to be 8 and we shall prove the following result.

TheOREM 1. Equation (1.1) with $r=d=b=1$ implies that

$$
\left(n, k, d_{1}, \ldots, d_{t}\right) \in\{(2,3,0,2),(1,4,0,1,3)\} .
$$

Theorem 1 answers a question of Erdös and Selfridge [5, p. 300]. Apart from the method of Erdős and Selfridge, the proof of Theorem 1 depends on the contributions of Wiles, Ribet and others on generalised Fermat equations. For equation (1.1) with $d=1, r>1$ and (1.2), we refer to Shorey [17] and Shorey and Nesterenko [21].

Now we suppose that equation (1.1) with $r=0$ and $d>1$ is satisfied. Marszałek [9] showed that $k$ is bounded by a number $c$ depending only on $d$. Saradha [15] proved that $c$ can be 3 for $d \in\{2,3,4,6\}$ and 4 for $d=5$. Shorey [18] showed that $c$ can be replaced by a number depending only on $P(d)$. Further, Shorey and Tijdeman [22] replaced $c$ by a number depending only on $\ell$ and $\omega(d)$ where $\omega(d)$ denotes the number of distinct prime divisors of $d$. Shorey and Tijdeman [22] also improved the result of Marszałek by 
proving that

$$
d \geq k^{C_{1} \log \log k}
$$

and Shorey [19] showed that

$$
n \geq k^{C_{2} \log \log k} \quad \text { for } \ell \geq 7 .
$$

The proof of the former inequality is elementary whereas the proof of the latter depends on Baker's theory of linear forms in logarithms, the best possible estimates of Shorey replacing $\log A_{1} \ldots \log A_{n}$ by $\log A_{n}$ for linear forms in logarithms with $\alpha_{i}$ 's very close to 1 and also on the irrationality measures of Baker by hypergeometric method.

It is clear from the proofs that the results in the previous paragraph are also valid for equation (1.1) with $r=1$ and $d>1$. Further, Saradha [15] proved that equation (1.1) with $r=1$ and $2 \leq d \leq 6$ is not valid for $k \geq 9$. For equation (1.1) with $r>1$ and $d>1$, we refer to Shorey and Tijdeman [24]. It has been conjectured by Erdös that equation (1.1) with $r=0$ and $d>1$ implies that $k$ is bounded by an absolute constant. Shorey [20] showed that the above conjecture for $\ell>3$ is a consequence of the $a b c$-conjecture and the proof depends on (1.3). For a survey of results on equation (1.1), we refer to [20] and [25].

We write

$$
d=D_{1} D_{2}
$$

where $D_{1}$ is the maximal divisor of $d$ such that all the prime divisors of $D_{1}$ are congruent to $1(\bmod \ell)$. Thus $D_{1}$ and $D_{2}$ are relatively prime positive integers such that $D_{2}$ has no prime divisor which is congruent to $1(\bmod \ell)$. Hence the assertion that $d$ has no prime divisor congruent to $1(\bmod \ell)$ is equivalent to $D_{1}=1$. Shorey [18] proved that equation (1.1) with $r=0$ and $d>1$ implies that

$$
D_{1}>1 \quad \text { if } k \geq C_{3} .
$$

The absolute constant $C_{3}$ turns out to be large in the proof of Shorey and we show that it can be replaced by 4 . More generally, we prove

THEOREM 2. Suppose that equation (1.1) with (1.2) is satisfied. Assume that

$$
k \geq 4 \quad \text { if } r=0 \quad \text { and } \quad k \geq 9 \quad \text { if } r=1 \text {. }
$$

Then $D_{1}>1$.

Thus, under the assumptions of Theorem 2, we see that $d$ is divisible by a prime congruent to $1(\bmod \ell)$ and hence $P(d) \geq 2 \ell+1$. Theorem 2 includes the results of Saradha stated above. The proof of Theorem 2 bases on the method of Erdös and Selfridge. We combine Theorem 2 with a result of Györy [7] that equation (1.1) with $r=0, k=3, P(b)<k$ and $d>1$ does not hold to obtain the following result. 
Corollary 1. Assume equation (1.1) with $r=0$ and $d>1$. Then $D_{1}>1$ if either $P(b)<k$ or $2 \mid d$.

It follows from Corollary 1 that equation (1.1) with $r=0$ and $d>1$ implies that $P(d) \geq 7$ if either $P(b)<k$ or $2 \mid d$. It is also clear from the proof of Theorem 2 that the assumption (1.6) in Theorem 2 can be relaxed to $k \geq 3$ if $r=0$ and $k \geq 4$ if $r=1$ whenever $d>1$ with $2 \mid d$ or $3 \mid d$.

Shorey and Tijdeman [22] sharpened (1.5) by showing that

$$
D_{1}>C_{4} k^{\ell-2} \text {. }
$$

The constant $C_{4}$ turns out to be small, and therefore the above inequality is trivial for small values of $k$. We obtain the following sharpening of (1.5), which improves the above estimate for small values of $k$. We put

$$
\theta= \begin{cases}1 & \text { if } \ell \nmid d \\ 1 / \ell & \text { if } \ell \mid d\end{cases}
$$

Then we prove

THEOREM 3. Suppose that equation (1.1) with (1.2) and (1.6) is satisfied. Then

$$
D_{1}>\alpha \theta k^{\beta}
$$

where $(\alpha, \beta)$ are as follows:

(i) $(1.59, \ell / 2-3-5 /(2 \ell))$ if $\ell \geq 17$,

(ii) $(1.1,43 / 13)$ if $\ell=13$,

(iii) $(.93,25 / 11)$ if $\ell=11$,

(iv) $(.73,9 / 7)$ if $\ell=7$,

(v) $(.6,7 / 5)$ if $\ell=5,5 \mid d$, $(.65,1 / 5)$ if $\ell=5,5 \nmid d$,

(vi) $(.41,1 / 3)$ if $\ell=3$.

Thus equation (1.1) with $r=0, d>1, k \geq 4$ and $\ell \geq 17$ implies that $d \geq D_{1} \geq 157$. Further, we observe that (1.1) with $d>1, k \geq 9$ and $\ell \geq 17$ implies that $d \geq D_{1} \geq 11993$. The computations in this paper have been carried out using MATHEMATICA. The proofs use several computational ideas. We thank Dr. C. Khare for useful discussions on Lemma 13.

2. Notation and preliminaries. We follow the notation of Section 1 throughout the paper. This is also the case with the notation which we introduce in this section. Let $q_{1}<q_{2}<\ldots$ be the sequence of all primes coprime to $d$. We write $\pi_{d}(k)$ for the number of all primes $\leq k$ and coprime to $d$. If $d=1$, we write $q_{i}=p_{i}$ for $i \geq 1$ and $\pi_{d}(k)=\pi(k)$. We shall use the estimates

$$
p_{i} \geq i \log i \quad \text { for } i \geq 1,
$$




$$
\pi_{d}(x) \leq \pi(x) \leq \frac{x}{\log x}+\frac{1.5 x}{\log ^{2} x} \quad \text { for } x>1
$$

and

$$
\pi(2 x)-\pi(x) \geq \frac{3 x}{5 \log x} \quad \text { for } x \geq 20.5,
$$

(see $[13$, p. 69]). Further we write

$$
\begin{array}{ll}
q_{i}(k)=q_{\pi_{d}(k)+i} & \text { for } i \geq 1, \\
p_{i}(k)=p_{\pi(k)+i} & \text { for } i \geq 1,
\end{array} \quad \chi= \begin{cases}2 & \text { if } r=0, d>1, k \geq 4, \\
1 & \text { otherwise }\end{cases}
$$

and

$$
\delta=\frac{n+(k-1) d}{k^{\ell+1}} .
$$

Further we put

$$
\alpha_{1}=\alpha_{1}(d, k)=(k-1) ! \prod_{p \mid d} p^{-\operatorname{ord}_{p}(k-1) !}
$$

$$
\begin{aligned}
\alpha_{2} & =\alpha_{2}(d, k, \ell, r)=1-\frac{\log \alpha_{1}}{k\left(\ell \log q_{\chi}(k)-\log k\right)}-\frac{\pi_{d}(k)+r+1}{k} \\
\alpha_{3} & =\alpha_{3}(d, k, \ell, r) \\
& =\frac{\left(\left[\alpha_{2} k\right]+1\right)}{k}\left(\frac{q_{1}(k)}{k}\right)^{\ell} \geq \frac{\left(\left[\alpha_{2} k\right]+1\right)}{k}\left(1+\frac{1}{k}\right)^{\ell} \geq \alpha_{2}
\end{aligned}
$$

We put

$$
\alpha_{4}=\alpha_{4}(d, k, \ell, r)=1-\frac{\log \alpha_{1}}{k\left(\ell \log k+\log \alpha_{3}\right)}-\frac{\pi_{d}(k)+r+1}{k} .
$$

For $0<\delta^{\prime}<1$, we put

$$
\begin{aligned}
\alpha_{5} & =\alpha_{5}(d, k, \ell, r) \\
& =\left(1-\delta^{\prime}-\frac{\log \alpha_{1}}{k\left(\ell \log k+\log \alpha_{3}\right)}-\frac{r+1}{k}\right)\left(\delta^{\prime} \log \left(\delta^{\prime} k\right)\right)^{\ell} .
\end{aligned}
$$

By (1.1), we write

(2.9) $\quad n+d_{i} d=a_{i} x_{i}^{\ell}, \quad P\left(a_{i}\right) \leq k, \quad a_{i}$ is $\ell$ th power free for $1 \leq i \leq t$, (2.10) $n+d_{i} d=A_{i} X_{i}^{\ell}, \quad P\left(A_{i}\right) \leq k, \quad \operatorname{gcd}\left(\prod_{p \leq k} p, X_{i}\right)=1 \quad$ for $1 \leq i \leq t$.

Let $S=\left\{A_{1}, \ldots, A_{t}\right\}, T=\left\{a_{1}, \ldots, a_{t}\right\}$ and set $|T|=t^{\prime}$. Let $d$ and $k$ be given. Let $m_{1} \geq 1, m_{2} \geq 0$ and $m_{3} \geq 0$ be integers such that $m_{1}+m_{2}+m_{3}=\pi_{d}(k)$ and

(2.11) $q_{1}<\ldots<q_{m_{1}}<q_{m_{1}+1}<\ldots<q_{m_{1}+m_{2}}<\ldots<q_{m_{1}+m_{2}+m_{3}} \leq k$.

Let $H\left(d, k, m_{1}, m_{2}\right)$ denote the number of $a_{i}$ 's in $T$ which are composed only of $q_{1}, \ldots, q_{m_{1}}$ and divisible by at most one of the primes $q_{m_{1}+1}, \ldots, q_{m_{1}+m_{2}}$ 
which divides at most to the first power. In particular, when $m_{2}=0$, $H\left(d, k, m_{1}, 0\right)$ represents the number of $a_{i}$ 's in $T$ which are composed only of $q_{1}, \ldots, q_{m_{1}}$. Suppose $\beta_{\mu}$ denotes the number of $a_{j}$ 's in $T$ divisible by $q_{\mu}$ and $\beta_{\mu \nu}$ denotes the number of $a_{j}$ 's in $T$ divisible by $q_{\mu} q_{\nu}$ for $1 \leq \mu, \nu \leq$ $m_{1}+m_{2}+m_{3}$. Then

$$
H\left(d, k, m_{1}, m_{2}\right) \geq t^{\prime}-\sum_{\mu=m_{1}+m_{2}+1}^{m_{1}+m_{2}+m_{3}} \beta_{\mu}-\sum_{m_{1}+1 \leq \mu, \nu \leq m_{1}+m_{2}} \beta_{\mu \nu} .
$$

In particular, when $m_{2}=0$, we have

$$
H\left(d, k, m_{1}, 0\right) \geq t^{\prime}-\sum_{\mu=m_{1}+1}^{m_{1}+m_{3}} \beta_{\mu} .
$$

We see from $(2.9)$ and $\operatorname{gcd}(n, d)=1$ that

$$
\beta_{\mu} \leq\left[\frac{k}{q_{\mu}}\right]+\varepsilon_{\mu}, \quad \beta_{\mu \nu} \leq\left[\frac{k}{q_{\mu} q_{\nu}}\right]+\varepsilon_{\mu \nu}
$$

where

$$
\varepsilon_{\mu}=\left\{\begin{array}{ll}
0 & \text { if } q_{\mu} \mid k \text { or } q_{\mu}>k, \\
1 & \text { if } q_{\mu} \nmid k,
\end{array} \quad \varepsilon_{\mu \nu}= \begin{cases}0 & \text { if } q_{\mu} q_{\nu} \mid k, \\
1 & \text { if } q_{\mu} q_{\nu} \nmid k .\end{cases}\right.
$$

By (2.12)-(2.14), we have

$$
\begin{aligned}
& H\left(d, k, m_{1}, m_{2}\right) \\
\geq & t^{\prime}-\sum_{\mu=m_{1}+m_{2}+1}^{m_{1}+m_{2}+m_{3}}\left(\left[\frac{k}{q_{\mu}}\right]+\varepsilon_{\mu}\right)-\sum_{m_{1}+1 \leq \mu, \nu \leq m_{1}+m_{2}}\left(\left[\frac{k}{q_{\mu} q_{\nu}}\right]+\varepsilon_{\mu \nu}\right) \\
:= & H_{0}\left(d, k, m_{1}, m_{2}\right)
\end{aligned}
$$

and

$$
H\left(d, k, m_{1}, 0\right) \geq t^{\prime}-\sum_{\mu=m_{1}+1}^{m_{1}+m_{3}}\left(\left[\frac{k}{q_{\mu}}\right]+\varepsilon_{\mu}\right)=H_{0}\left(d, k, m_{1}, 0\right) .
$$

It follows immediately from the definition of $H_{0}\left(d, k, m_{1}, m_{2}\right)$ that

$$
H_{0}\left(d, k, m_{1}, m_{2}\right) \geq H_{0}\left(1, k, m_{1}, m_{2}\right) .
$$

Further, we notice that whenever $t^{\prime}=t$ we have

$$
H_{0}(1, k, m, 0)=f_{0}(k, m)
$$

and

$$
H_{0}\left(1, k, m_{1}, m_{2}\right) \geq F_{0}\left(k, m_{1}, m_{2}\right)
$$

if $m_{1}=\pi\left(k^{3}\right)$ and $m_{2}=\pi\left(k^{5}\right)-\pi\left(k^{3}\right)$ where $f_{0}(k, m)$ and $F_{0}\left(k, m_{1}, m_{2}\right)$ are given in $[15,(19),(22)]$. In $[15,(35)$, Table 2], lower bounds, say $X$, for $f_{0}(k, m)$ with $4 \leq k \leq 2238$ and $F_{0}^{*}\left(m_{1}, m_{2}\right)$ for $F_{0}\left(k, m_{1}, m_{2}\right)$ with $2239 \leq k \leq 11379$ are given. We shall give below the table of $[15,(35)$, 
Table 2] which will be used later. We observe here that for $k \geq 9$, the value $X$ has been calculated taking $r=1$. Therefore, when we consider the case $r=0$ and $k \geq 9$, we may replace $X$ by $X+1$.

$$
\begin{aligned}
& f_{0}(k, 2) \geq 4, \quad 4 \leq k \leq 22 ; \quad f_{0}(k, 3) \geq 8, \quad 23 \leq k \leq 102 ; \\
& f_{0}(k, 4) \geq 16, \quad 103 \leq k \leq 282 ; \quad f_{0}(k, 5) \geq 22, \quad 283 \leq k \leq 612 ; \\
& f_{0}(k, 6) \geq 38, \quad 613 \leq k \leq 1102 ; \quad f_{0}(k, 7) \geq 66, \quad 1103 \leq k \leq 1636 ; \\
& f_{0}(k, 8) \geq 115, \quad 1637 \leq k \leq 2238 \text {. }
\end{aligned}
$$

Table 1

\begin{tabular}{cccc}
\hline$m_{1}$ & $m_{2}$ & $k$ & $F_{0}^{*}\left(m_{1}, m_{2}\right)$ \\
\hline 4 & 11 & $2239-2808$ & 112 \\
4 & 12 & $2809-2960$ & 121 \\
5 & 11 & $2961-3480$ & 195 \\
5 & 12 & $3481-3720$ & 210 \\
5 & 13 & $3721-4488$ & 226 \\
5 & 14 & $4489-5040$ & 241 \\
5 & 15 & $5041-5165$ & 257 \\
6 & 14 & $5166-5328$ & 418 \\
6 & 15 & $5329-6240$ & 445 \\
6 & 16 & $6241-6888$ & 472 \\
6 & 17 & $6889-7920$ & 499 \\
6 & 18 & $7921-9408$ & 526 \\
6 & 19 & $9409-10200$ & 553 \\
6 & 20 & $10201-10608$ & 580 \\
6 & 21 & $10609-11379$ & 607 \\
\hline
\end{tabular}

Finally, we conclude this section with the following fundamental result of Erdős (see [5, Lemma 2]).

Lemma 1. For $1 \leq i \leq t$, let $n+d_{i} d=B_{i} B_{i}^{\prime}$, where $B_{i}$ and $B_{i}^{\prime}$ are positive integers such that $P\left(B_{i}\right) \leq k$. Let $\mathcal{S}=\left\{B_{1}, \ldots, B_{t}\right\}$. For every prime $p \leq k$ with $\operatorname{gcd}(p, d)=1$, choose $B_{i_{p}} \in \mathcal{S}$ such that $p$ does not appear to a higher power in the factorisation of any other element of $\mathcal{S}$. Let $\mathcal{S}_{1}$ be the subset of $\mathcal{S}$ obtained by deleting from $\mathcal{S}$ all $B_{i_{p}}$ with $p \leq k$ and $\operatorname{gcd}(p, d)=1$. Then

$$
\prod_{B_{i} \in \mathcal{S}_{1}} B_{i} \leq \alpha_{1} .
$$

Proof. This follows by observing that the above product divides

$$
\prod_{\substack{p<k \\ \operatorname{cd}(p, d)=1}} p^{\left[\frac{k-1}{p}\right]+\left[\frac{k-1}{p^{2}}\right]+\ldots} \text {. - }
$$

We shall apply Lemma 1 several times. Besides, we refer to Lemma 1 for its argument at many places. 
3. A sharpening of a theorem of Sylvester. A well known theorem of Sylvester [26] states that a product of $k$ consecutive positive integers $>k$ is divisible by a prime exceeding $k$. Sylvester [26] extended his result by proving that $P(\Delta)>k$ if $n \geq d+k$ and $r=0$. Further, Langevin [8] relaxed the assumption $n \geq d+k$ to $n>k$. Finally, Shorey and Tijdeman [23] proved that $P(\Delta) \leq k$ and $r=0$ imply that $(n, d, k)=(2,7,3)$. This is an immediate consequence of the following analogous result for $r=1$.

Theorem 4. Let $r=1, d>1$ and $k \geq 4$. Suppose that

$$
P(\Delta) \leq k \text {. }
$$

Then

$$
\begin{aligned}
\left(n, d, k, d_{1}, \ldots, d_{t}\right) \in\{ & (1,5,4,0,1,3),(2,7,4,0,1,2),(3,5,4,0,1,3), \\
& (1,2,5,0,1,2,4),(2,7,5,0,1,2,4) \\
& (4,7,5,0,2,3,4),(4,23,5,0,1,2,4)\} .
\end{aligned}
$$

We refer to Section 1 for the definition of $\Delta$ and we recall that $\operatorname{gcd}(n, d)$ $=1$. The proof of Theorem 4 depends on Lemma 1 and estimate (2.2) from prime number theory. We do not need the results on an analogue of Bertrand's postulate for certain arithmetic progressions as is the case in [23]. Finally, we observe that Theorem 4 is equivalent to the following sharpening of the result of Shorey and Tijdeman stated in this section and it is of independent interest.

Theorem $4^{\prime}$. Let $r=0, d>1$ and $k \geq 4$. Suppose that

$$
\begin{aligned}
& (n, d, k) \\
& \quad \notin\{(1,5,4),(2,7,4),(3,5,4),(1,2,5),(2,7,5),(4,7,5),(4,23,5)\} .
\end{aligned}
$$

Then $\Delta$ is divisible by at least 2 distinct primes exceeding $k$.

We observe that the assumption (3.2) is necessary in Theorem $4^{\prime}$.

Proof of Theorem 4. We first show that (3.1) implies $k \geq 9$. Let $k=4$. There are three terms composed only of 2 and 3 . Out of these terms we delete one in which 2 appears to a maximal power and one in which 3 appears to a maximal power to see that either $n+d \leq 6$ or $n \leq 6$ such that at least one of $P(n(n+i d)(n+j d)) \leq 3$ with $1 \leq i<j \leq 3$. Suppose that $n+d \leq 6$. Let $n=1, d=2$. Then $P(n+(k-1) d)=7, P(n+(k-2) d)=5$, which contradicts (3.1). All cases other than $\left(n, d, k, d_{1}, \ldots, d_{t}\right)=(1,5,4,0,1,3)$ and $(3,5,4,0,1,3)$ are excluded similarly. Now let $n \leq 6$ with at least one of $P(n(n+i d)(n+j d)) \leq 3$ with $1 \leq i<j \leq 3$. By the case $k=3$ of [23], we need to consider only the cases $P(n(n+d)(n+3 d)) \leq 3$ and $P(n(n+2 d)(n+3 d)) \leq 3$. Let $P(n(n+d)(n+3 d)) \leq 3$. Then $n$ and $d$ are odd. If $n=1$, then $1+3 d=2^{\alpha}, 1+d=2 \cdot 3^{\beta}$, and if $n=3$, then $3+3 d=2 \cdot 3^{\beta}, 3+d=2^{\alpha}$. This implies that $n=1, d=5$ or $n=3, d=5$ 
by the well known result on the Catalan equation that 9 and 8 are the only powers of 3 and 2, respectively, that differ by 1 . The details for the other case are similar. Let $k=5$. By a similar argument, we show that (3.1) is not possible unless

$\left(n, d, k, d_{1}, \ldots, d_{t}\right)$

$$
=(1,2,5,0,1,2,4),(2,7,5,0,1,2,4),(4,7,5,0,2,3,4),(4,23,5,0,1,2,4) .
$$

Let $k \in\{6,7,8\}$. Then we have $n \leq 12$ and $n+d \leq 60$. For these values of $n$ and $d$, we check that (3.1) is contradicted.

Thus we may suppose that $k \geq 9$ from now onward. By (3.1), we see that $P\left(n+d_{i} d\right) \leq k$ for $1 \leq i \leq t$. Hence by Lemma 1 with $B_{i}=n+d_{i} d, B_{i}^{\prime}=1$, we have

$$
\prod_{i=0}^{k-\pi_{d}(k)-2}(n+i d) \leq \alpha_{1}
$$

On the other hand,

$$
\prod_{i=0}^{k-\pi_{d}(k)-2}(n+i d) \geq\left\{\begin{array}{l}
\left(k-\pi_{d}(k)-2\right) ! d^{k-\pi_{d}(k)-2} \\
\left(k-\pi_{d}(k)-1\right) ! d^{k-\pi_{d}(k)-1}
\end{array} \text { if } n \geq d .\right.
$$

Therefore

$$
\begin{aligned}
& d^{k-\pi_{d}(k)-2} \leq(k-1) \ldots\left(k-\pi_{d}(k)-1\right) \prod_{p \mid d} p^{-\operatorname{ord}_{p}(k-1) !} \\
& d^{k-\pi_{d}(k)-1} \leq(k-1) \ldots\left(k-\pi_{d}(k)\right) \prod_{p \mid d} p^{-\operatorname{ord}_{p}(k-1) !} \quad \text { if } n \geq d .
\end{aligned}
$$

By using $\pi_{d}(k) \leq \pi(k)$, we see that

$$
d^{k-\pi(k)-2} \leq(k-1) \ldots(k-\pi(k)-1) .
$$

By (3.3), we have

$$
d<k^{\left(\pi_{d}(k)+1\right) /\left(k-\pi_{d}(k)-2\right)} \prod_{p \mid d} p^{-\left(\operatorname{ord}_{p}(k-1) !\right) /\left(k-\pi_{d}(k)-2\right)} .
$$

Thus by (2.2),

$$
d<\exp \left(\frac{1+\frac{1.5}{\log k}+\frac{\log k}{k}}{1-\frac{1}{\log k}-\frac{1.5}{\log ^{2} k}-\frac{2}{k}}\right) \prod_{p \mid d} p^{-\left(\frac{k-p-1}{p-1}-\frac{\log (k-1)}{\log p}\right) /(k-2)},
$$

which implies that

$$
d<\exp \left(\frac{1+\frac{1.5}{\log k}+\frac{\log k}{k}}{1-\frac{1}{\log k}-\frac{1.5}{\log ^{2} k}-\frac{2}{k}}\right) .
$$

First we claim that $d<4$ for $k \geq 25$. Suppose $d \geq 4$ and $k \geq 25$. Then by (3.8), we have $k \leq 3615$. Further we see from (3.8) that $d \leq 9$ for $k \geq 67$. 
By (3.5), we check that $d \leq 9$ for every $k$ with $25 \leq k \leq 66$. Thus $4 \leq d \leq 9$. In fact $k \leq 91$ if $d=4$ by (3.7) and we use (3.8) to get $k \leq 399$ if $d=5$; $k \leq 171$ if $d=6 ; k \leq 108$ if $d=7 ; k \leq 81$ if $d=8$; and $k \leq 66$ if $d=9$. Now we use (3.3) to show that this is not possible and our claim is proved.

Let $d \geq 4$. Then $9 \leq k \leq 24$. Now we use (3.5) and (3.3) to get $d=5, k \in$ $\{13,19\} ; d=11, k \in\{9,10\} ; d=13, k \in\{9,11\} ; d=17, k \in\{9,11,13\} ; d=$ $19, k \in\{11,13\}$. Further we use (3.4) to conclude that $n<d$. For these values of $n, d$ and $k$, we check that at least two of $P(n+(k-i) d)$ with $1 \leq i \leq 7$ exceed $k$, which contradicts (3.1). Thus $d<4$.

Let $d=3$. Then $k \leq 357$ by (3.7), $k \leq 24$ by (3.3) and $n<3$ by (3.4) and we check that (3.1) is contradicted in these cases.

Let $d=2$. Then $k \leq 3757$ by (3.7). We reduce the value of $k$ to 118 by using (3.3) with exact value of $\pi_{d}(k)$ and $\operatorname{ord}_{2}(k-1)$ !. Further, we use (3.4) to derive that $n=1$ for $9 \leq k \leq 118$ unless $k \in\{19,23,24,31,32,47\}$. By (3.1), we have

$$
\pi(n+2 k-2) \leq \pi(n-1)+\pi(k)+1,
$$

which implies that $n>1$. Therefore, we conclude that $k \in\{19,23,24,31$, $32,47\}$. If $n \geq 10$, then (3.4) can be sharpened to

$$
d^{k-\pi_{d}(k)-1} \leq 4 !\left(k-\pi_{d}(k)+4\right) \ldots(k-1) 2^{-\operatorname{ord}_{2}(k-1) !},
$$

which is not possible for the above values of $k$. Thus $n \leq 9$. Finally, we check that (3.9) does not hold for $n \leq 9$ and $k \in\{19,23,24,31,32,47\}$.

4. Lemmas for the proof of Theorem 2. In this section, we shall always assume equation (1.1) with (1.2). We recall that $k \geq 4$ if $r=1, d>1$ and the tuples $\left(n, d, k, d_{1}, \ldots, d_{t}\right)$ given in Theorem 4 are excluded. Further, equation (1.1) with $r=0$ is not satisfied for tuples $(n, d, k)$ appearing in Theorem $4^{\prime}$. Hence we derive from Theorems 4 and $4^{\prime}$ that

$$
n+(k-1) d \geq\left(q_{\chi}(k)\right)^{\ell} \geq(k+1)^{\ell},
$$

which implies that

$$
\delta \geq \frac{\left(q_{\chi}(k)\right)^{\ell}}{k^{\ell+1}} \geq \frac{1}{k}\left(1+\frac{1}{k}\right)^{\ell} .
$$

Now we use (4.1) to show the distinctness of $a_{i}$ 's and $A_{j}$ 's.

Lemma 2. Suppose that equation (1.1) with (1.2) is satisfied. (a) If

$$
D_{1} \leq \frac{\ell \theta\left(q_{\chi}(k)\right)^{\ell-1}}{k^{2-1 / \ell}}
$$

then at least $t-1$ elements of each of the sets $S$ and $T$ are distinct. 
(b) If

$$
D_{1} \leq \frac{\theta\left(q_{\chi}(k)\right)^{\ell-1}}{k^{2-1 / \ell}}
$$

then the elements of each of the sets $S$ and $T$ are distinct.

Proof. (a) Let $T^{\prime}=T-\left\{a_{0}\right\}$. We show that the elements of $T^{\prime}$ are distinct. Suppose $a_{i}=a_{j}$ for some $a_{i}, a_{j} \in T^{\prime}$ with $i \neq j$. Then we see from (2.9) that

$$
\begin{aligned}
\left(d_{i}-d_{j}\right) d & =\left(n+d_{i} d\right)-\left(n+d_{j} d\right) \\
& =a_{j}\left(x_{i}^{\ell}-x_{j}^{\ell}\right)=a_{j}\left(\frac{x_{i}^{\ell}-x_{j}^{\ell}}{x_{i}-x_{j}}\right)\left(x_{i}-x_{j}\right) .
\end{aligned}
$$

We assume without loss of generality that $x_{i}>x_{j}$. We observe that all the prime divisors of $\left(x_{i}^{\ell}-x_{j}^{\ell}\right) /\left(x_{i}-x_{j}\right)$ are congruent to $1(\bmod \ell)$ except possibly $\ell$ which appears only to the first power if and only if $\ell \mid\left(x_{i}-x_{j}\right)$. Now we see from $(4.5),(1.4)$ and (1.7) that $\theta D_{2}$ divides $x_{i}-x_{j} \operatorname{since} \operatorname{gcd}\left(a_{i}, d\right)=1$. Thus $x_{i}-x_{j} \geq \theta D_{2}$ and

$$
k d>\left(d_{i}-d_{j}\right) d \geq a_{j} \ell \theta D_{2} x_{j}^{\ell-1} .
$$

Since $j \neq 0$, we have $a_{j} x_{j}^{\ell} \geq n+d$. Thus

$$
k D_{1}>\ell \theta\left(a_{j} x_{j}^{\ell}\right)^{(\ell-1) / \ell} \geq \ell \theta(n+d)^{(\ell-1) / \ell}>\ell \theta\left(\frac{1}{k}(n+(k-1) d)\right)^{(\ell-1) / \ell} .
$$

Now we use (4.1) to get $D_{1}>\left(\ell \theta\left(q_{\chi}(k)\right)^{\ell-1}\right) / k^{2-1 / \ell}$, which contradicts (4.3). Hence the elements of $T^{\prime}$ are distinct, which implies the assertion of Lemma 2(a). The proof that at least $t-1$ elements of $S$ are distinct is similar.

(b) We show that the elements of $T$ are distinct. We proceed as in (a). Assuming $a_{i}=a_{j}$ for some $a_{i}, a_{j} \in T$ with $i \neq j$, we get (4.5). Further $x_{i}>x_{j}$ implies that $n+d_{i} d>n+d_{j} d$. Hence $n+d_{i} d \geq n+d$. Thus from (4.5) we get

$$
k d>\left(d_{i}-d_{j}\right) d \geq a_{i} \theta D_{2} x_{i}^{\ell-1},
$$

which implies that

$$
k D_{1}>\theta\left(a_{i} x_{i}^{\ell}\right)^{(\ell-1) / \ell} \geq \theta(n+d)^{(\ell-1) / \ell} .
$$

Now as in Lemma 2(a), we obtain a contradiction implying $|T|=t$. The proof for $|S|=t$ is similar.

We apply Lemma 2 to improve the bound for $\delta$ in (4.2).

Lemma 3. Suppose that equation (1.1) with (1.2) and (4.4) is satisfied. Then

(i) $\delta \geq \alpha_{3}$,

(ii) $\delta \geq\left(q_{\left(\left[\alpha_{4} k\right]+1\right)}(k)\right)^{\ell} / k^{\ell+1}$,

(iii) $\delta \geq \alpha_{5}$ if $0<\delta^{\prime}<1$ is such that $\delta^{\prime} k \geq \pi_{d}(k)$. 
Proof. (i) Suppose that equation (1.1) with (1.2) and (4.4) is satisfied. Then $|S|=t$ by Lemma 2(b). Let $S_{1}$ be the set of all $A_{i} \in S$ for which $X_{i}=1$ and write $S_{2}$ for the complement of $S_{1}$ in $S$. Put $\left|S_{1}\right|=h$. By Lemma 1 , there exists a subset $S_{3}$ of $S_{1}$ such that $\left|S_{3}\right| \geq h-\pi_{d}(k)$ and

$$
\prod_{A_{i} \in S_{3}} A_{i} \leq \alpha_{1} .
$$

Now for any $A_{i} \in S_{3}$ with $i \neq 0$, we see that $A_{i} \geq n+d$. Thus

$$
\prod_{A_{i} \in S_{3}} A_{i} \geq(n+d)^{h-\pi_{d}(k)-1} \text {. }
$$

Since

$$
n+d>\frac{1}{k}(n+(k-1) d) \geq \frac{\left(q_{\chi}(k)\right)^{\ell}}{k}
$$

by (4.1), we get

$$
\prod_{A_{i} \in S_{3}} A_{i}>\left(\frac{\left(q_{\chi}(k)\right)^{\ell}}{k}\right)^{h-\pi_{d}(k)-1} .
$$

Combining the above estimates for $\prod_{A_{i} \in S_{3}} A_{i}$, we have

$$
h<\frac{\log \alpha_{1}}{\ell \log q_{\chi}(k)-\log k}+\pi_{d}(k)+1 .
$$

Therefore we derive from Lemma 2(b) and $t=k-r$ that

$$
\left|S_{2}\right|>k-r-\frac{\log \alpha_{1}}{\ell \log q_{\chi}(k)-\log k}-\pi_{d}(k)-1=\alpha_{2} k,
$$

which implies that $\left|S_{2}\right| \geq\left[\alpha_{2} k\right]+1$. Now we arrange the $A_{i}$ 's belonging to $S_{2}$ in increasing order. Further we observe from (2.10) that $X_{i} \geq q_{1}(k)$ if $A_{i} \in S_{2}$. Hence

$$
n+(k-1) d \geq\left(\left[\alpha_{2} k\right]+1\right)\left(q_{1}(k)\right)^{\ell}=\alpha_{3} k^{\ell+1} .
$$

Now (i) follows from (4.6) and (2.4).

(ii) We apply (4.6) in place of (4.1) to derive that $n+d>\alpha_{3} k^{\ell}$ and

$$
h<\frac{\log \alpha_{1}}{\ell \log k+\log \alpha_{3}}+\pi_{d}(k)+1,
$$

which implies that $\left|S_{2}\right|>\alpha_{4} k$. We observe from (2.10) and $\operatorname{gcd}(n, d)=1$ that $X_{i}$ 's with $A_{i} \in S_{2}$ are pairwise coprime with all prime factors exceeding $k$ and coprime to $d$. Consider the set $S_{4}$ of all prime factors of $X_{i}$ 's with $A_{i} \in S_{2}$. Then $\left|S_{4}\right| \geq\left|S_{2}\right|>\alpha_{4} k$. Therefore the largest element of $S_{4}$ is at least $q_{\left(\left[\alpha_{4} k\right]+1\right)}(k)$. Now the assertion of Lemma 3(ii) follows immediately from (2.4) and (2.10).

(iii) We may suppose that the $X_{i}$ 's corresponding to the $A_{i}$ 's in $S_{2}$ are primes, otherwise the assertion of Lemma 3(iii) follows from (2.10) and 
$0<\delta^{\prime}<1$. As seen in (ii), $\left|S_{2}\right|>\alpha_{4} k$. Further these primes are distinct. We first arrange the $X_{i}$ 's in increasing order, say $X_{1}<\ldots<X_{\left[\alpha_{4} k\right]+1}<\ldots$ We omit $X_{1}, \ldots, X_{\nu}$ where $\nu=\left[\delta^{\prime} k-\pi_{d}(k)\right] \geq 0$ by our assumption. We may assume that $\nu \leq\left[\alpha_{4} k\right]$, otherwise the assertion is trivial. Then we are left with $X_{\nu+1}<\ldots<X_{\left[\alpha_{4} k\right]+1}<\ldots$ Here each $X_{i} \geq q_{\nu+1}(k)$. Now we take the corresponding $A_{i}$ 's and arrange them in increasing order. Then we conclude from Lemma $2,(2.10)$ and (2.1) that

$$
\begin{aligned}
n+(k-1) d & \geq\left(\alpha_{4} k-\nu\right)\left(q_{\nu+1}(k)\right)^{\ell} \geq\left(\alpha_{4} k-\nu\right) p_{\pi(k)+\nu+1}^{\ell} \\
& \geq\left(\alpha_{4} k-\nu\right)(\pi(k)+\nu+1)^{\ell} \log ^{\ell}(\pi(k)+\nu+1),
\end{aligned}
$$

which implies the assertion.

The next result is due to Erdős and Selfridge [5] and it is fundamental in their method.

Lemma 4. Let $1 \leq \ell^{\prime} \leq \ell-1$. Suppose that equation (1.1) with $d=1$ and (1.2) is satisfied. Then for no distinct $\ell^{\prime}$-tuples $\left(i_{1}, \ldots, i_{\ell^{\prime}}\right)$ and $\left(j_{1}, \ldots, j_{\ell^{\prime}}\right)$ with $i_{1} \leq \ldots \leq i_{\ell^{\prime}}$ and $j_{1} \leq \ldots \leq j_{\ell^{\prime}}$, the ratio of the two products $a_{i_{1}} \ldots a_{i_{\ell^{\prime}}}$ and $a_{j_{1}} \ldots a_{j_{\ell^{\prime}}}$ is an $\ell$ th power of a rational number.

For a proof of Lemma 4, see also Saradha [15, Lemma 4]. The following result extends and improves Lemma 4 considerably.

Lemma 5. Let $1 \leq \ell^{\prime} \leq \ell-1, \kappa>0$ and

$$
\kappa_{0}=\min \left(\frac{\ell}{\ell^{\prime}(\kappa+1)^{\left(\ell-\ell^{\prime}\right) / \ell}}, \frac{\kappa}{(\kappa+1) \ell^{\prime 1 / \ell}}\right) .
$$

Assume equation (1.1) with (1.2) and

$$
D_{1} \leq \kappa_{0} \theta \delta^{\left(\ell-\ell^{\prime}\right) / \ell} k^{\ell-\ell^{\prime}-\ell^{\prime} / \ell} .
$$

Then the assertion of Lemma 4 is valid.

Proof. Let $\left(i_{1}, \ldots, i_{\ell^{\prime}}\right)$ and $\left(j_{1}, \ldots, j_{\ell^{\prime}}\right)$ with $i_{1} \leq \ldots \leq i_{\ell^{\prime}}$ and $j_{1} \leq \ldots \leq$ $j_{\ell^{\prime}}$ be distinct $\ell^{\prime}$-tuples. Suppose that

$$
a_{i_{1}} \ldots a_{i_{\ell^{\prime}}}=a_{j_{1}} \ldots a_{j_{\ell^{\prime}}}\left(t_{1} / t_{2}\right)^{\ell}
$$

where $t_{1}$ and $t_{2}$ are positive integers. There is no loss of generality in assuming that $t_{1}$ and $t_{2}$ are relatively prime. We claim

$$
\left(n+d_{i_{1}} d\right) \ldots\left(n+d_{i_{\ell^{\prime}}} d\right) \neq\left(n+d_{j_{1}} d\right) \ldots\left(n+d_{j_{\ell^{\prime}}} d\right) .
$$

Suppose

$$
\left(n+d_{i_{1}} d\right) \ldots\left(n+d_{i_{\ell^{\prime}}} d\right)=\left(n+d_{j_{1}} d\right) \ldots\left(n+d_{j_{\ell^{\prime}}} d\right) .
$$

Then we cancel any term on the left hand side which equals some term on the right hand side. Thus there exists at least one term different from $n$ say, $n+d_{i_{1}} d$, on the left hand side. Since $\operatorname{gcd}(n, d)=1$, we have $n+d \leq n+d_{i_{1}} d \leq \operatorname{gcd}\left(n+d_{i_{1}} d, n+d_{j_{1}} d\right) \ldots \operatorname{gcd}\left(n+d_{i_{1}} d, n+d_{j_{\ell^{\prime}}} d\right) \leq k^{\ell^{\prime}}$. 
Thus $n+(k-1) d<k(n+d) \leq k^{\ell}$, which contradicts (4.1). This proves (4.9). Now we may assume without loss of generality that the left hand side of (4.9) is greater than the right hand side. By (2.9) and (4.9), we have

$$
\begin{aligned}
\left(n+d_{i_{1}} d\right) \ldots(n+ & \left.d_{i_{\ell^{\prime}}} d\right)-\left(n+d_{j_{1}} d\right) \ldots\left(n+d_{j_{\ell^{\prime}}} d\right) \\
& =a_{i_{1}} \ldots a_{i_{\ell^{\prime}}} x_{i_{1}}^{\ell} \ldots x_{i_{\ell^{\prime}}}^{\ell}-a_{j_{1}} \ldots a_{j_{\ell^{\prime}}} x_{j_{1}}^{\ell} \ldots x_{j_{\ell^{\prime}}}^{\ell} \\
& =\frac{a_{j_{1}} \ldots a_{j_{\ell^{\prime}}}}{t_{2}^{\ell}}\left(\left(t_{1} x_{i_{1}} \ldots x_{i_{\ell^{\prime}}}\right)^{\ell}-\left(t_{2} x_{j_{1}} \ldots x_{j_{\ell^{\prime}}}\right)^{\ell}\right) \\
& =\frac{a_{j_{1}} \ldots a_{j_{\ell^{\prime}}}}{t_{2}^{\ell}}\left(x^{\ell}-y^{\ell}\right)
\end{aligned}
$$

where $x=t_{1} x_{i_{1}} \ldots x_{i_{\ell^{\prime}}}$ and $y=t_{2} x_{j_{1}} \ldots x_{{\ell^{\prime}}^{\prime}}$. We see from (4.8) that $a_{i_{1}} \ldots a_{i_{\ell^{\prime}}} / t_{1}^{\ell}$ and $a_{j_{1}} \ldots a_{j_{\ell^{\prime}}} / t_{2}^{\ell}$ are positive integers since $\operatorname{gcd}\left(t_{1}, t_{2}\right)=1$. We put $A=a_{j_{1}} \ldots a_{j_{\ell^{\prime}}} / t_{2}^{\ell}$. Further we observe that the left hand side of (4.10) is divisible by $d$ and the first factor on the right hand side is relatively prime to $d$ since $\operatorname{gcd}(n, d)=1$. Therefore we see from (1.4) and (1.7) that $\theta D_{2}$ divides $x-y$. Hence $x \geq y+\theta D_{2}$ and the left hand side of (4.10) is

$$
\begin{aligned}
& \geq A\left(\left(\begin{array}{l}
\ell \\
1
\end{array}\right) \theta D_{2} y^{\ell-1}+\left(\begin{array}{l}
\ell \\
2
\end{array}\right)\left(\theta D_{2}\right)^{2} y^{\ell-2}+\ldots+\left(\theta D_{2}\right)^{\ell}\right) \\
& \geq\left(\begin{array}{l}
\ell \\
1
\end{array}\right) \theta D_{2}\left(A y^{\ell}\right)^{(\ell-1) / \ell}+\left(\begin{array}{l}
\ell \\
2
\end{array}\right)\left(\theta D_{2}\right)^{2}\left(A y^{\ell}\right)^{(\ell-2) / \ell}+\ldots+\left(\theta D_{2}\right)^{\ell} \\
& \geq\left(\begin{array}{l}
\ell \\
1
\end{array}\right) \theta D_{2} n^{\ell^{\prime}(\ell-1) / \ell}+\left(\begin{array}{l}
\ell \\
2
\end{array}\right)\left(\theta D_{2}\right)^{2} n^{\ell^{\prime}(\ell-2) / \ell}+\ldots+\left(\theta D_{2}\right)^{\ell},
\end{aligned}
$$

since $A y^{\ell}=\left(a_{j_{1}} x_{j_{1}}^{\ell}\right) \ldots\left(a_{j_{\ell^{\prime}}} x_{j_{\ell^{\prime}}}^{\ell}\right) \geq n^{\ell^{\prime}}$. On the other hand, the left hand side of (4.10) is

$$
<\left(\begin{array}{c}
\ell^{\prime} \\
1
\end{array}\right) k d n^{\ell^{\prime}-1}+\left(\begin{array}{c}
\ell^{\prime} \\
2
\end{array}\right)(k d)^{2} n^{\ell^{\prime}-2}+\ldots+(k d)^{\ell^{\prime}} .
$$

By comparing the above two estimates, we obtain

$$
\begin{aligned}
& \left\{\left(\begin{array}{l}
\ell \\
1
\end{array}\right) \theta D_{2} n^{\ell^{\prime}(\ell-1) / \ell}-\left(\begin{array}{c}
\ell^{\prime} \\
1
\end{array}\right) k d n^{\ell^{\prime}-1}\right\} \\
& +\left\{\left(\begin{array}{l}
\ell \\
2
\end{array}\right)\left(\theta D_{2}\right)^{2} n^{\ell^{\prime}(\ell-2) / \ell}-\left(\begin{array}{c}
\ell^{\prime} \\
2
\end{array}\right)(k d)^{2} n^{\ell^{\prime}-2}\right\} \\
& +\ldots+\left\{\left(\begin{array}{l}
\ell \\
\ell^{\prime}
\end{array}\right)\left(\theta D_{2}\right)^{\ell^{\prime}} n^{\ell^{\prime}\left(\ell-\ell^{\prime}\right) / \ell}-(k d)^{\ell^{\prime}}\right\}+\ldots+\left(\theta D_{2}\right)^{\ell}<0 .
\end{aligned}
$$

Now we divide the proof into two cases. 
CASE (i). Let $n>\frac{\delta}{\kappa+1} k^{\ell+1}$. For $1 \leq i \leq \ell^{\prime}$, the $i$ th term in the curly bracket of (4.11) is

$$
\begin{aligned}
\left(\begin{array}{c}
\ell \\
i
\end{array}\right) & \left(\theta D_{2}\right)^{i} n^{\ell^{\prime}(\ell-i) / \ell}-\left(\begin{array}{c}
\ell^{\prime} \\
i
\end{array}\right)(k d)^{i} n^{\ell^{\prime}-i} \\
= & n^{\ell^{\prime}-i} D_{2}^{i}\left\{\left(\begin{array}{c}
\ell \\
i
\end{array}\right) \theta^{i} n^{i\left(\ell-\ell^{\prime}\right) / \ell}-\left(\begin{array}{c}
\ell^{\prime} \\
i
\end{array}\right) k^{i} D_{1}^{i}\right\} \\
& >n^{\ell^{\prime}-i} D_{2}^{i}\left\{\left(\begin{array}{c}
\ell \\
i
\end{array}\right) \theta^{i}\left(\frac{\delta}{\kappa+1}\right)^{i\left(\ell-\ell^{\prime}\right) / \ell} k^{i(\ell+1)\left(\ell-\ell^{\prime}\right) / \ell}-\left(\begin{array}{c}
\ell^{\prime} \\
i
\end{array}\right) k^{i} D_{1}^{i}\right\} \\
& >0
\end{aligned}
$$

since

$$
D_{1} \leq \frac{\ell \theta \delta^{\left(\ell-\ell^{\prime}\right) / \ell} k^{\ell-\ell^{\prime}-\ell^{\prime} / \ell}}{\ell^{\prime}(\kappa+1)^{\left(\ell-\ell^{\prime}\right) / \ell}}
$$

by (4.7). This contradicts (4.11).

CASE (ii). Let

$$
n \leq \frac{\delta}{\kappa+1} k^{\ell+1} .
$$

Then by (2.4),

$$
d \geq \frac{\delta \kappa}{\kappa+1} k^{\ell}
$$

From (4.11), it follows that there exists an $i$ with $1 \leq i \leq \ell^{\prime}$ such that

$$
\left(\begin{array}{c}
\ell \\
i
\end{array}\right)\left(\theta D_{2}\right)^{i} n^{\ell^{\prime}(\ell-i) / \ell}-\left(\begin{array}{c}
\ell^{\prime} \\
i
\end{array}\right)(k d)^{i} n^{\ell^{\prime}-i}<-\frac{\left(\theta D_{2}\right)^{\ell}}{\ell^{\prime}} .
$$

This implies that

$$
\left(\begin{array}{c}
\ell^{\prime} \\
i
\end{array}\right)(k d)^{i} n^{\ell^{\prime}-i}>\frac{\left(\theta D_{2}\right)^{\ell}}{\ell^{\prime}} .
$$

Then, by (1.4), we have

$$
D_{1}^{\ell}>\frac{\theta^{\ell} d^{\ell-i}}{\ell^{\prime}\left(\begin{array}{c}
\ell^{\prime} \\
i
\end{array}\right) k^{i} n^{\ell^{\prime}-i}} .
$$

Now we apply (4.12) and (4.13) to obtain

$$
D_{1}>\frac{\kappa \theta \delta^{\left(\ell-\ell^{\prime}\right) / \ell} k^{\ell-\ell^{\prime}-\ell^{\prime} / \ell}}{(\kappa+1)^{1-\ell^{\prime} / \ell} \ell^{\prime 1 / \ell}\left(\kappa^{i}\left(\begin{array}{c}
\ell^{\prime} \\
i
\end{array}\right)\right)^{1 / \ell}}>\frac{\kappa \theta \delta^{\left(\ell-\ell^{\prime}\right) / \ell} k^{\ell-\ell^{\prime}-\ell^{\prime} / \ell}}{(\kappa+1) \ell^{\prime 1 / \ell}},
$$

which contradicts (4.7).

The next result is an immediate extension of an estimate of Erdős and Selfridge [5].

Lemma 6. Let $1 \leq \ell^{\prime} \leq \ell-1$ and assume equation (1.1) with (1.2). Suppose that for no distinct $\ell^{\prime}$-tuples $\left(i_{1}, \ldots, i_{\ell^{\prime}}\right)$ and $\left(j_{1}, \ldots, j_{\ell^{\prime}}\right)$ with $i_{1} \leq$ 
$\ldots \leq i_{\ell^{\prime}}$ and $j_{1} \leq \ldots \leq j_{\ell^{\prime}}$, the ratio of two products $a_{i_{1}} \ldots a_{i_{\ell^{\prime}}}$ and $a_{j_{1}} \ldots a_{j_{\ell^{\prime}}}$ is an $\ell$ th power of a rational number. Then

$$
\left(\begin{array}{c}
H\left(d, k, m_{1}, m_{2}\right)+\ell^{\prime}-1 \\
\ell^{\prime}
\end{array}\right) \leq \ell^{m_{1}}\left(\begin{array}{c}
\ell^{\prime}+m_{2} \\
\ell^{\prime}
\end{array}\right)
$$

where the left hand side is equal to zero if $H\left(d, k, m_{1}, m_{2}\right)<1$.

Let $g=g(\ell)=(\ell-5) / 2$ and $\ell^{\prime}=\ell-g$. Then we see by induction that if (4.14) does not hold for some odd $\ell=\ell_{1}$, then it does not hold for every odd $\ell>\ell_{1}$ provided that

$$
H\left(d, k, m_{1}, m_{2}\right)>\left(1+\frac{2}{\ell_{1}}\right)^{m_{1}}\left(m_{2}+\frac{\ell_{1}+7}{2}\right)-\frac{l_{1}+5}{2} .
$$

We apply Lemma 5 to derive

Lemma 7. Equation (1.1) with (1.2) and

$$
D_{1} \leq \theta k^{\ell-3+1 / \ell}
$$

implies that $k<11380$.

Proof. Let $k \geq 11380$. We observe from (4.16) and (4.1) that (4.4) is valid. Hence Lemma 3 holds. Now we use $\log \alpha_{1}<k \log k$ by (2.5), $\ell \geq 3$, $k \geq 11380, r \leq 1, q_{\chi}(k)>k$ and $(2.2)$ to find $\alpha_{3} \geq .3754$. Further, we compute $\alpha_{5}$ with $\delta^{\prime}=1 / 2$ to get $\alpha_{5} \geq 12.4678$. Hence by Lemma 3(iii), $\delta \geq 12.4678$. Now we take $\ell^{\prime}=2$ and $\kappa=5$ in Lemma 5 . We see that $\delta^{(\ell-2) / \ell} \geq \delta^{1 / 3} \geq 2.3187$. We observe that $\ell /\left(2(\kappa+1)^{(\ell-2) / \ell}\right)$ is an increasing function of $\ell$. Thus we get $\kappa_{0} \geq .6614$. Hence the right hand side of inequality (4.7) is $>1.5335 \cdot \theta k^{\ell-2-2 / \ell}$. Thus (4.16) implies that (4.7) is satisfied. Hence we conclude by Lemma 5 that the products $a_{i} a_{j}$ for $1 \leq i, j \leq t$ are distinct. Then all the estimates of [15, Lemma 8] are valid and these estimates yield $k<11380$ as in [15, pp. 165-166]. This is a contradiction.

Lemma 8. Suppose that equation (1.1) with (1.2), (1.6) and $\ell \geq 17$ is satisfied. Then

$$
D_{1}>1.59 \cdot \theta k^{\ell / 2-3-5 /(2 \ell)} .
$$

Proof. Suppose that

$$
D_{1} \leq 1.59 \cdot \theta k^{\ell / 2-3-5 /(2 \ell)} .
$$

Then (4.4) holds. Hence Lemma 3 is valid. For $k \geq 30$, we use $\ell \geq 17, r \leq 1$ in (2.7) and in (2.8) with $\delta^{\prime}=1 / 2$ to get $\alpha_{5} \geq 64.5882$. Thus $\delta \geq 64.5882$ for $k \geq 30$ by Lemma 3(iii). Further, we see from Lemma 3(ii) that $\delta \geq$ 11.1022 for $4 \leq k \leq 29$ by calculating $q_{\left[\alpha_{4} k\right]+1}(k)$. Hence $\delta \geq 11.1022$ for $k \geq 4$. Further we take $\ell^{\prime}=(\ell+5) / 2$ and $\kappa=3.65$. Then we observe that $(2 \ell) /(\ell+5)(\kappa+1)^{(\ell-5) /(2 \ell)}$ is an increasing function of $\ell$. Therefore we find 
that $\kappa_{0} \geq .6816$. Thus

$$
\kappa_{0} \theta \delta^{\left(\ell-\ell^{\prime}\right) / \ell} k^{\ell-\ell^{\prime}-\ell^{\prime} / \ell}>1.5940 \cdot \theta k^{\ell / 2-3-5 /(2 \ell)} .
$$

Thus we see from (4.18) that $D_{1}$ satisfies (4.7) and (4.16). Therefore by Lemma 7 , we have $k<11380$ and by Lemmas 5 and 6 ,

$$
\left(\begin{array}{c}
H\left(d, k, m_{1}, m_{2}\right)+\frac{\ell+5}{2}-1 \\
\frac{\ell+5}{2}
\end{array}\right) \leq \ell^{m_{1}}\left(\begin{array}{c}
\frac{\ell+5}{2}+m_{2} \\
\frac{\ell+5}{2}
\end{array}\right) .
$$

Let $H\left(d, k, m_{1}, m_{2}\right) \geq Y$. We wish to contradict (4.19) for suitable values of $m_{1}, m_{2}$ and $\ell$. For this, it is enough to show that

$$
\left(\begin{array}{c}
Y+\frac{\ell+5}{2}-1 \\
\frac{\ell+5}{2}
\end{array}\right)>\ell^{m_{1}}\left(\begin{array}{c}
\frac{\ell+5}{2}+m_{2} \\
\frac{\ell+5}{2}
\end{array}\right)
$$

for suitable values of $m_{1}, m_{2}$ and $\ell$. Let $k \leq 2238$ and $m_{1}=m, m_{2}=0$. By (2.15)-(2.17) and (1.6), we can take for $Y$ the values given as lower bounds for $f_{0}(k, m)$ in (2.17) to satisfy (4.20) with $\ell=17$. Let $2238<k<11380$. By (2.15), (2.16) and (2.18), we can take $Y$ as $F_{0}^{*}\left(m_{1}, m_{2}\right)$ given by Table 1 to satisfy (4.20) with $\ell=17$. Further we check that

$$
Y \geq\left(1+\frac{2}{\ell_{1}}\right)^{m_{1}}\left(m_{2}+\frac{\ell_{1}+7}{2}\right)-\frac{\ell_{1}+5}{2}
$$

for $\ell_{1}=17$ and for suitable values of $m_{1}, m_{2}$ mentioned above. Consequently, inequality (4.20) is satisfied for all odd $\ell \geq 17$ and for suitable values of $m_{1}, m_{2}$ given above.

Lemma 9. Suppose that equation (1.1) with (1.2), $3 \leq \ell \leq 13$ and (4.16) is satisfied. Then

$$
D_{1}>.7 \theta \delta^{\beta_{1}} k^{\beta_{2}}
$$

where $\left(\beta_{1}, \beta_{2}\right)$ are given as follows:

(i) $(4 / 13,43 / 13)$ if $\ell=13$,

(ii) $(3 / 11,25 / 11)$ if $\ell=11$,

(iii) $(2 / 7,9 / 7)$ if $\ell=7$,

(iv) $(2 / 5,7 / 5)$ if $\ell=5,5 \mid d, k \neq 4$, $(1 / 5,1 / 5)$ if $\ell=5,5 \mid d, k=4$ or $\ell=5,5 \nmid d$,

(v) $(1 / 3,1 / 3)$ if $\ell=3$.

Proof. We derive from Lemma 7 that $k<11380$. We follow the argument of Lemma 8 . We take $\ell^{\prime}=9$ for $\ell=13$; $\ell^{\prime}=8$ for $\ell=11 ; \ell^{\prime}=5$ for $\ell=7$; $\ell^{\prime}=3$ for $\ell=5,5 \mid d, k \neq 4 ; \ell^{\prime}=4$ for $\ell=5,5 \mid d, k=4$ or $\ell=5,5 \nmid d ; \ell^{\prime}=2$ for $\ell=3$. We take $\kappa=8$ for $\ell \neq 5 ; \kappa=7$ if $\ell=5,5 \mid d, k \neq 4$; and $\kappa=15$ if $\ell=5,5 \mid d, k=4$ or if $\ell=5,5 \nmid d$ in the expression for $\kappa_{0}$ in Lemma 5 to find that $\kappa_{0} \geq .7$. Suppose

$$
D_{1} \leq .7 \theta \delta^{\beta_{1}} k^{\beta_{2}}
$$


Then

$$
D_{1} \leq \kappa_{0} \theta \delta^{\beta_{1}} k^{\beta_{2}}
$$

Also (4.22) with (i) to (v) implies that (4.7) is satisfied. Therefore the assertion of Lemma 5 is valid. Hence we conclude that (4.14) holds. We also note that $a_{i}$ 's are distinct. For all the cases except $\ell=5,5 \mid d, k \neq 4$, we use (1.6), (2.19) and Table 1 as in the proof of Lemma 8 to contradict (4.14). Now we consider the case $\ell=5,5 \mid d, k \neq 4$. In this case $\ell^{\prime}=3$ and $k \geq 5$. Since $5 \mid d$, we observe that $H\left(d, k, m_{1}, 0\right) \geq f_{0}\left(k, m_{1}+1\right)$ whenever $m_{1} \geq 2$. Now we use (2.19) and Table 1 as in Lemma 8 to contradict (4.14) whenever $k \geq 23$. Further we use the above inequality to calculate $H(d, k, 2,0) \geq 5$ for $k \leq 22$, which contradicts (4.14), implying the assertion of Lemma 9.

We conclude this section by stating a result of Győry [7].

LEMMA 10. Equation (1.1) with $r=0, k=3, P(b)<k$ and $d>1$ does not hold.

5. Proof of Theorem 3. We assume equation (1.1) with (1.2) and (1.6). We may suppose that (4.16) is satisfied, otherwise the assertion of Theorem 3 follows immediately. We observe that (1.8) with (i) is the assertion of Lemma 8. Thus we may suppose that $3 \leq \ell \leq 13$. Then by Lemma 9 , (4.21) holds with (i) to (v). Let $\ell=13$. Then $\beta_{1}=4 / 13$ and $\delta^{4 / 13} \geq 1.5936$ for $4 \leq k \leq 30$ by Lemma 3(ii) and for $k \geq 31$ by Lemma 3(iii). Now the assertion for $\ell=13$ follows from (4.21). Similarly we have $\delta^{\beta_{1}} \geq$ $1.3382 ; 1.0514 ; .8663 ; .9307 ; .5849$ according as $\ell=11 ; \ell=7 ; \ell=5,5 \mid \bar{d}$; $\ell=5,5 \nmid d ; \ell=3$, respectively. Finally we apply (4.21) to complete the proof of Theorem 3.

6. Proof of Theorem 2. We suppose that equation (1.1) with (1.2), (1.6) and $D_{1}=1$ is satisfied. Now we derive from Theorem 3 that $3 \leq \ell \leq 7$. Then from Lemma 9 we get

$$
1>.7 \theta \delta^{\beta_{1}} k^{\beta_{2}}
$$

with $\left(\beta_{1}, \beta_{2}\right)$ as in Lemma 9 . Let $\ell=7$. Then by Theorem 3 , we get $7 \mid d$ and $k=4,5$. We see from (6.1) that $\delta \leq 2.2628$ if $k=5$ and $\delta \leq 6.1764$ if $k=4$. On the other hand, we derive from (4.2) and (1.6) that $\delta \geq 160$. This is a contradiction.

Let $\ell=5$ and $5 \nmid d$. Then we derive from (1.8) and (6.1) that $k \leq 8$ and $\delta \leq 5.95 / k$. Thus $r=0$ by (1.6) and $k=4,5$ by Lemma 3(ii). Now we see from (4.2) and (1.2) that $d=1$ and $P(\Delta)=5$ if $k=4, P(\Delta)=7$ if $k=5$. Thus $n+k-1 \geq 5^{5}$. On the other hand, we see from an argument of Lemma 1 that $n \leq 12$. This is a contradiction. 
Let $\ell=5$ and $5 \mid d$. Then we derive from (1.8) and (6.1) that $k=4$ and $n+(k-1) d<\left(\frac{200}{7}\right)^{5}$. If $3 \mid d$, the assertion follows from Lemma 10 . If $2 \mid d$, there are two successive terms which are $\ell$ th powers, contradicting $D_{1}=1$. Thus $2 \nmid d, 3 \nmid d$. Since $D_{1}=1$, we see that $\operatorname{gcd}(p, d)=1$ whenever $p \in\{11,31,41,61,71\}$. By an argument of Lemma 1 , we find $i, j$ with $0 \leq$ $i<j \leq 3$ such that $A_{i}, A_{j} \in\{1,2,3,4,6\}$ and $X_{i}, X_{j}$ are primes between 7 and 23 . We have $d=\left(A_{j} X_{j}^{\ell}-A_{i} X_{i}^{\ell}\right) / h$ with $h=j-i$. We calculate all possible pairs $\left(A_{i} X_{i}^{\ell}, d\right)$ satisfying the above conditions. Further we check that $A_{i} X_{i}^{\ell}+2 d$ if $h=1, A_{i} X_{i}^{\ell}+3 d$ if $h=2$, and $A_{i} X_{i}^{\ell}+2 d$ if $h=3$ is divisible by a prime $>7$ to a power which is $\not \equiv 0(\bmod \ell)$. Hence $k \neq 4$.

Let $\ell=3$ and $3 \nmid d$. Then by (1.8), we see that $k \leq 14$. Further $\delta \leq$ $2.9155 / k$ by (6.1), which contradicts Lemma 3(ii) when $4 \leq k \leq 14$.

Let $\ell=3$ and $3 \mid d$. By (1.8) and (6.1), we get $k \leq 391$ and

$$
\delta<\left(\frac{30}{7}\right)^{3} \frac{1}{k} .
$$

For $k \geq 102$, we see from Lemma 3 (iii) with $\delta^{\prime}=1 / 2$ that $\delta \geq \alpha_{5} \geq .7779$, contradicting (6.2). For every $k$ with $71 \leq k \leq 102$, we give a lower bound for $\alpha_{5}$ by using $\pi_{d}(k) \leq \pi(k)$ and the exact value of $\pi(k)$ to contradict (6.2). Thus $k \leq 70$. We see from Lemma 2 that $|T|=t$ unless $k \in\{10,12,16,18\}$ in which case $|T|=t-1$. Since $a_{i}$ 's are cube free, we observe that $H(d, k, 1,0)$ $\leq 3$ and $H(d, k, 2,0) \leq 9$. By using $\operatorname{gcd}\left(a_{i}, 3\right)=1$ for $1 \leq i \leq t$, we calculate that $H(d, k, 2,0) \geq f_{0}(k, 2) \geq 10$ for $26 \leq k \leq 70$ and $H(d, k, 1,0) \geq$ $f_{0}(k, 1) \geq 4$ for $4 \leq k \leq 25, k \notin\{12,16,18,23,24\}$. Let $k=24$. Then $f_{0}(k, 1) \geq 3$ and hence $H(d, k, 1,0)=3$. This implies that the number of $a_{i}$ 's divisible by the primes $5,7,11,13,17,19$ and 23 is $5,4,3,2,2,2$ and 2, respectively. Each of these $a_{i}$ 's is divisible precisely by one of these primes. Therefore 23 divides $a_{0}, a_{23}$ and 7 divides $a_{1}, a_{8}, a_{15}, a_{22}$. Hence 5 divides either $a_{2}, a_{7}, a_{12}, a_{17}, a_{22}$ or $a_{3}, a_{8}, a_{13}, a_{18}, a_{23}$. This is a contradiction since $7 \mid a_{22}$ and $23 \mid a_{23}$. The cases $k=12,16,18,23$ are excluded similarly.

Proof of Corollary 1. By Theorem 2, we may assume that $k \leq 3$. If $k=3$ and $P(b)<k$, the assertion follows from Lemma 10. If $k=3$ and $2 \mid d$, then there are at least two distinct $a_{i}, a_{j}$ which are equal to one. Thus $\left|x_{i}^{\ell}-x_{j}^{\ell}\right|=d$ or $2 d$ and this is not possible since $D_{1}=1$. If $k=2$ and $P(b)<k$ or $2 \mid d$, then $b=1$, implying $x_{1}^{\ell}-x_{0}^{\ell}=d$, which is not possible again by $D_{1}=1$.

7. Additional lemmas for the proof of Theorem 1. The proof of Theorem 1 depends on Lemmas 4 and 6 of Section 4 . In this section, we give the other lemmas required for the proof of Theorem 1. We start with the following result on a generalised Fermat equation. 
Lemma 11. Let $\ell \geq 3$ and $\alpha$ an integer with $1 \leq \alpha<\ell$. Then the equation

$$
x^{\ell}+y^{\ell}=2^{\alpha} z^{\ell}
$$

in non-zero relatively prime integers $x, y, z$ has no solution for $\alpha>1$, and for $\alpha=1$ the equation has only the trivial solution for which $x y z= \pm 1$.

The above assertion for $\alpha>1$ is due to Ribet [11] and for $\alpha=1$ to Darmon and Merel [2]. Lemma 10 and the following result of Györy are consequences of Lemma 11.

Lemma 12. Equation (1.1) with $r=0, d=1, k=3$ and (1.2) has no solution.

In fact, as in Sander [14], the contributions of Wiles, Ribet and others give the following result on a more general Fermat equation than (7.1).

Lemma 13. Let $\ell \geq 5$. Let $a, b, c$ be non-zero integers such that either $P(a b c) \leq 3$ or $a, b, c$ are composed of only 2 and 5 . Then the equation

$$
\begin{array}{ll}
a x^{\ell}-b y^{\ell}=c z^{\ell} & \text { in non-zero integers } x, y, z \\
& \text { with } \operatorname{gcd}\left(a x^{\ell}, b y^{\ell}, c z^{\ell}\right)=1, \operatorname{ord}_{2}\left(b y^{\ell}\right) \geq 4
\end{array}
$$

has no solution.

Proof. We follow the arguments given in Ribet [11]. Let $x, y, z$ be nonzero integers satisfying (7.2). We put $A=a x^{\ell}, B=-b y^{\ell}$ and $C=c z^{\ell}$. Thus $A+B=C$. We assume that $A \equiv-1(\bmod 4)$ by possibly multiplying the given equation by -1 . Further we observe that $\operatorname{gcd}(A, B)=1$ and $16 \mid B$. We form the Frey elliptic curve $E$ :

$$
Y^{2}=X(X-A)(X+B)
$$

Then the conductor $N_{E}$ of $E$ is given by

$$
N_{E}=2^{t} \operatorname{rad}^{\prime}(A B C)
$$

where $t$ is a non-negative integer and $\operatorname{rad}^{\prime}(A B C)$ means the product of odd prime divisors of $A B C$. According to the computations made by Diamond and Kramer,

$$
t= \begin{cases}0 & \text { if } \operatorname{ord}_{2}\left(b y^{\ell}\right)=4, \\ 1 & \text { if } \operatorname{ord}_{2}\left(b y^{\ell}\right)>4 .\end{cases}
$$

Thus $E$ is a semistable elliptic curve and hence by the works of Wiles and Wiles and Taylor, it is modular. Hence there exists a cusp form of weight 2 and level $N_{E}$. Further we see that if $\Delta_{E}$ denotes the minimal discriminant of $E$, then

$$
\Delta_{E}=2^{u}(A B C)^{2}
$$

where $u$ is an integer. Thus for any $p \mid \Delta_{E}$ we have

$$
\operatorname{ord}_{p}\left(\Delta_{E}\right) \equiv 0(\bmod \ell)
$$


except for $p=2,3$ if $P(a b c) \leq 3$ and except for $p=2,5$ if $a$ and $b$ are composed of only 2 and 5 . Hence by a theorem of Ribet, there is a cusp form of weight 2 and level 2 or 3 or 6 if $P(a b c) \leq 3$ or there is a cusp form of weight 2 and level 2 or 5 or 10 if $a$ and $b$ are composed only of 2 and 5 . We see from the dimension formula given in [16, pp. 23-25] that $X_{0}(2), X_{0}(3), X_{0}(5), X_{0}(6), X_{0}(10)$ are of dimension 0 . This is a contradiction.

Bennett [1] developed hypergeometric methods to show

Lemma 14. If $a, b$ and $\ell$ are integers with $a b \neq 0$ and $\ell \geq 3$, then the equation

$$
\left|a x^{\ell}-b y^{\ell}\right|=1
$$

has at most one solution in positive integers $(x, y)$. Hence the equation

$$
\left|(a+1) x^{\ell}-a y^{\ell}\right|=1
$$

has the only solution $(x, y)=(1,1)$.

Finally, we show that the assertion of Theorem 1 is valid when $P(\Delta) \leq k$.

Lemma 15. Equation (1.1) with $r=b=d=1$ implies that $P(\Delta)>k$ unless

$$
\left(n, k, d_{1}, \ldots, d_{t}\right) \in\{(2,3,0,2),(1,4,0,1,3)\} .
$$

Proof. Assume equation (1.1) with $r=b=d=1$ and $P(\Delta) \leq k$. Let $k=3$. Then $n(n+2)=y^{\ell}$ with either $n=2^{\alpha} 3^{\beta}, n+2=2^{\gamma}$ or $n=2^{\alpha}, n+2=2^{\gamma} 3^{\beta}$ where $\beta \equiv 0(\bmod \ell)$. Now we use a result on the Catalan equation stated in Section 3 to see that $n=2$. We show that $k \geq 17$. Let $4 \leq k \leq 16$. Then we see by an argument of Lemma 1 that $n \leq 12$ if $4 \leq k \leq 8$ and $n \leq 72$ if $9 \leq k \leq 16$. Now we check that

$$
\pi(n+k-1)-\pi(n-1) \geq 2
$$

for these values of $n$ and $k$ except when $k=4, n \in\{6,7,8,9,12\} ; k=5$, $n \in\{6,8,12\} ; k=9, n \in\{20,32,44,48,49,50,62\} ; k=10, n \in\{48,49\}$; $k=11, n=48$, which are excluded since $\Delta$ is an $\ell$ th power. Therefore we may suppose that (7.3) holds. This implies that there exists a prime $p \geq n$ dividing $\Delta$. Let $n \geq 11$. Then $\operatorname{ord}_{p}(\Delta) \leq 2$ since $n+k-1 \leq 87$ and $p \geq 11$. Finally, we check that $\Delta$ is not an $\ell$ th power for $n \leq 10$ and $4 \leq k \leq 16$. Thus $k \geq 17$.

Suppose $n<k$. Then $(n+k-1) / 2 \geq n$. Now using (2.3) we check that

$$
\pi(n+k-1)-\pi\left(\frac{n+k-1}{2}\right) \geq 2 \quad \text { if } n+k-1 \geq 41 .
$$

This means that if $n+k-1 \geq 41$, there exists a prime $p>(n+k-1) / 2$ dividing $\Delta$ and $\operatorname{ord}_{p}(\Delta)=1$. Thus we may assume that $n+k-1 \leq 40$. 
These values of $n$ and $k$ are excluded since $\Delta$ is an $\ell$ th power. Thus we may suppose that $n \geq k$. By Lemma 1 , we have

$$
n^{k-\pi(k)-1} \leq k^{k}
$$

i.e.

$$
k \leq \frac{k \log k}{\log n}+\pi(k)+1 .
$$

Suppose $n+i_{0}$ is the omitted term. Let $0 \leq i_{0} \leq[k / 2]$. By a result of Sylvester, there exists a prime $p \geq k / 2$ dividing the product $(n+[k / 2]+1)$ $\ldots(n+k-1)$. This prime $p$ can divide at most two terms of the product $\Delta$. Hence by equation (1.1) with $r=b=d=1$ we have

$$
n+k-1 \geq\left(\frac{k}{2}\right)^{\ell-1} .
$$

Suppose now $[k / 2]<i_{0} \leq k-1$. Since $n>k$, we again apply the result of Sylvester to $n(n+1) \ldots(n+[k / 2])$ and find that $(7.5)$ is satisfied. Thus (7.5) always holds. By combining (7.4) and (7.5), we have

$$
k \leq \frac{k \log k}{\log \left(\left(\frac{k}{2}\right)^{\ell-1}-k+1\right)}+\pi(k)+1,
$$

from which we derive that $k \leq 33$ since $\ell \geq 3$. Further we see from (7.4) that $n \leq 280$. Also we observe that $n \notin\{17,18,110,114,200,201,202,203,204$, $205,206\}$ since $\Delta$ is an $\ell$ th power. These values of $n$ and $k$ are excluded as earlier by checking (7.3) when $n \geq 19$ and the proof of Lemma 15 is complete since $n>k$.

8. Proof of Theorem 1. Suppose that equation (1.1) with $r=b=d=$ 1 holds. Then we may assume (1.2) by Lemma 15. Now we conclude that $k \leq 8$ by Saradha [15, Theorem 1(a)] or Theorem 2. Further the assertions of Lemmas 4 and 6 are valid. In particular, the $a_{i}$ 's are distinct. Let $k=8$. Then $f_{0}(k, 2) \geq 3$. Further we apply Lemmas 4 and 6 with $\ell^{\prime}=\ell-1, m_{1}=2$, $m_{2}=0$ as in the proof of Lemma 8 to conclude that $f_{0}(k, 2)=3$. We write the terms of the left hand side of equation (1.1) as in (2.9). Then 7 divides $a_{0}$ and $a_{7}, 5$ divides $a_{1}$ and $a_{6}$ and by Lemma 12 , the omitted term is either $n+3$ or $n+4$. Thus we have two possible equations:

$$
n(n+1)(n+2)(n+4)(n+5)(n+6)(n+7)=y^{\ell}
$$

or

$$
n(n+1)(n+2)(n+3)(n+5)(n+6)(n+7)=y^{\ell} .
$$

We prove the assertion of Theorem 1 for (8.1). The proof for (8.2) is similar. We assume without reference that (8.1) holds in the case $k=8$. Suppose no 
$a_{i}$ equals 1 . Then we observe that $6 \mid(n+2)$ and we write $(2.9)$ as

$$
\begin{gathered}
n=2^{f_{0}} 7^{i_{0}} x_{0}^{\ell}, \quad n+1=5^{h_{1}} x_{1}^{\ell}, \quad n+2=2^{f_{2}} 3^{g_{2}} x_{2}^{\ell}, \quad n+4=2^{f_{4}} x_{4}^{\ell}, \\
n+5=3^{g_{5}} x_{5}^{\ell}, \quad n+6=2^{f_{6}} 5^{h_{6}} x_{6}^{\ell}, \quad n+7=7^{i_{7}} x_{7}^{\ell} .
\end{gathered}
$$

Here all the indices are non-negative integers $<\ell$ such that $i_{0}, h_{1}, f_{4}, g_{5}, h_{6}, i_{7}$ are positive and either $f_{2}$ or $g_{2}$ is non-zero. Suppose $f_{4} \geq 2$. Then $f_{2}=f_{6}=1$, which implies $a_{2} a_{5}=a_{1} a_{6}\left(\frac{3}{5}\right)^{\ell}$. This contradicts Lemma 4. Thus $f_{4}=1$. Suppose $f_{2} \geq 3$. Then $f_{6}=2$, giving $a_{1} a_{6}=a_{4} a_{4} 5^{\ell}$, a contradiction. If $f_{2}=2$, then $a_{2} a_{5}=a_{4} a_{4} 3^{\ell}$ gives a contradiction. Let $f_{2}=1$. Then $f_{6}=1$ or 2. If $f_{6}=1$, then $a_{1} a_{6}=a_{2} a_{5}\left(\frac{5}{3}\right)^{\ell}$, and if $f_{6}=2$, then $a_{1} a_{6}=a_{4} a_{4} 5^{\ell}$. These give the necessary contradiction. If $f_{2}=0$, then $x_{2}$ is even, say $2 x_{2}^{\prime}$, and we get $x_{4}^{\ell}-2^{\ell-1} 3^{g_{2}} x_{2}^{\prime \ell}=1$. This equation has no solution for $\ell \geq 5$ by Lemma 13. Thus we may assume for $\ell \geq 5$ that one of the $a_{i}$ 's, say $a_{j_{0}}$, is 1 . We note that $j_{0} \in\{2,4,5\}$. Then

$$
a_{0} a_{1} \ldots a_{j_{0}-1} a_{j_{0}+1} \ldots a_{7}=a_{j_{0}} \ldots a_{j_{0}} h^{\ell}
$$

for some positive integer $h$. Here, in the product on the right hand side, $a_{j_{0}}$ is taken 6 times. This is a contradiction to Lemma 4 with $\ell^{\prime}=6$ if $\ell \geq 7$.

Now we turn to considering the case $\ell=5$. First, we suppose $n+2=x_{2}^{5}$. Then $6 \nmid(n+4)$ and $6 \nmid(n+5)$. So $n+4=2^{f_{4}} x_{4}^{5}$ or $3^{g_{4}} x_{4}^{5}$ where $f_{4}>0$, $g_{4}>0$. If $n+4=2^{f_{4}} x_{4}^{5}$, then $2 \mid x_{2}$. Hence $f_{4}=1$. Then $x_{4}^{5}-16 x_{2}^{\prime 5}=1$ where $x_{2}=2 x_{2}^{\prime}$. This is not possible by Lemma 11. Suppose $n+4=3^{g_{4}} x_{4}^{5}$. Then $n+5=2^{f_{5}} x_{5}^{5}$. As 3 divides $n+1$ and $n+7$, we see that $g_{4}$ is either 1 or 3 . Further $n+2=x_{2}^{5}$ implies that $n+4 \equiv 1,2,3(\bmod 11)$ and $n+5 \equiv 2,3,4(\bmod 11)$. Hence $n+4=3 x_{4}^{5}$ and $n+5 \equiv 4(\bmod 11)$. Therefore $n+5=4 x_{5}^{5}$. Thus $4 x_{5}^{5}-3 x_{4}^{5}=1$. By Lemma 14, this equation has the only solution $\left(x_{4}, x_{5}\right)=(1,1)$, which is not possible. Secondly, we consider the case when $n+4=x_{4}^{5}$. Then $n+2=3^{g_{2}} x_{2}^{5}$ or $2 \cdot 3^{g_{2}} x_{2}^{5}$. Let $n+2=3^{g_{2}} x_{2}^{5}$. Since $n+4 \equiv 0,1,10(\bmod 11)$, we have $g_{2}=1$ or 2 . If $g_{2}=2$, then $g_{5}=3$, which implies $9 \mid 3$, a contradiction. If $g_{2}=1$, then $n$ is odd and we have

$$
\begin{gathered}
n=7^{i_{0}} x_{0}^{5}, \quad n+1=2^{f_{1}} 5^{h_{1}} x_{1}^{5}, \quad n+2=3 x_{2}^{5}, \quad n+4=x_{4}^{5}, \\
n+5=2^{f_{5}} 3^{4} x_{5}^{5}, \quad n+6=5^{h_{6}} x_{6}^{5}, \quad n+7=2^{f_{7}} 7^{i_{7}} x_{7}^{5} .
\end{gathered}
$$

Here $f_{1}, f_{5}, f_{7}$ are positive satisfying $f_{1}+f_{5}+f_{7}=5$, which implies $\left(f_{1}, f_{5}, f_{7}\right)$ $=(2,2,1)$ or $(1,1,3)$. Then we see that $a_{1} a_{6}=a_{2} a_{5}\left(\frac{5}{3}\right)^{5}$, contradicting Lemma 4. Suppose $n+2=2 \cdot 3^{g_{2}} x_{2}^{5}$. Then $x_{4}$ is even. If $g_{2}>0$, then $n+1=5^{h_{1}} x_{1}^{5}, n+5=3^{g_{5}} x_{5}^{5}$ and $n+6=2 \cdot 5^{h_{6}} x_{6}^{5}$. Thus $a_{1} a_{6}=a_{2} a_{5}\left(\frac{5}{3}\right)^{5}$. This is a contradiction. If $g_{2}=0$, then $16 x_{4}^{\prime 5}-x_{2}^{5}=1$ with $x_{4}=2 x_{4}^{\prime}$, contradicting Lemma 11. Finally, we take $n+5=x_{5}^{5}$. If $3 \mid a_{2}$, then $n+4=$ $2^{f_{4}} x_{4}^{5}$ and $x_{5}^{5}-2^{f_{4}} x_{4}^{5}=1$, contradicting Lemma 11 . Thus $3 \nmid a_{2}$. We again apply Lemma 11 to $(n+5)-(n+4)$ to see that $3 \mid a_{4}$. Also we have $f_{2}>0$. 
Thus

$$
\begin{gathered}
n=2^{f_{0}} 7^{i_{0}} x_{0}^{5}, \quad n+1=3^{g_{1}} 5^{h_{1}} x_{1}^{5}, \quad n+2=2^{f_{2}} x_{2}^{5}, \\
n+4=2^{f_{4}} 3^{g_{4}} x_{4}^{5}, \quad n+5=x_{5}^{5}, \quad n+6=2^{f_{6}} 5^{h_{6}} x_{6}^{5}, \quad n+7=3^{g_{7}} 7^{i_{7}} x_{7}^{5} .
\end{gathered}
$$

Now $g_{1}+g_{4}+g_{7}=5$, which implies that $\left(g_{1}, g_{4}, g_{7}\right)$ is a permutation of $(3,1,1)$. Similarly, by counting the power of 2 in $a_{i}$ 's, we conclude that $\left(f_{0}, f_{2}, f_{4}, f_{6}\right)$ is a permutation of $(2,1,1,1)$. When $\left(f_{0}, f_{2}, f_{4}, f_{6}\right)=(2,1,1,1)$, we have $x_{4}$ even, say $2 x_{4}^{\prime}$, and $2^{5} 3^{g_{4}} x_{5}^{15}-x_{2}^{5}=1$. This is impossible by Lemma 13. The cases $\left(f_{0}, f_{2}, f_{4}, f_{6}\right)=(1,2,1,1)$ and $(1,1,1,2)$ are excluded similarly. Let $\left(f_{0}, f_{2}, f_{4}, f_{6}\right)=(1,1,2,1)$. If $\left(g_{1}, g_{4}, g_{7}\right)=(3,1,1),(1,3,1)$ and $(1,1,3)$, then $a_{0} a_{2} a_{7}=a_{4} a_{5} a_{5} 7^{5}, a_{0} a_{7}=a_{1} a_{6}\left(\frac{7}{5}\right)^{5}$ and $a_{1} a_{2} a_{6}=a_{4} a_{5} a_{5} 5^{5}$ respectively, a contradiction to Lemma 4 . The other possibilities are excluded similarly. Thus $\ell \neq 5$.

Let $\ell=3$. Then $a_{i}$ 's are cube-free. Since $n \equiv 0(\bmod 7)$ and $a_{i} \in$ $\{1,2,3,4,6,9,12,18,36\}$, we see that $a_{2}, a_{5} \in\{2,9,12\}$ and $a_{4} \in\{3,4,18\}$. Let $a_{2}=2$. Then $a_{5}=9$ and $9 x_{5}^{3}-2 x_{2}^{3}=3$, which is not possible. The other cases are excluded similarly. This completes the proof of Theorem 1 for $k=8$.

Let $k=6$. Arguing as at the beginning of the case $k=8$, we find that we have either

$$
n(n+1)(n+2)(n+4)(n+5)=y^{\ell}
$$

or

$$
n(n+1)(n+3)(n+4)(n+5)=y^{\ell}
$$

with $5 \mid n$. Further we see that if $\ell \geq 5$, the proof is similar to the case $k=8$ and $\ell \geq 7$. Therefore we assume that $\ell=3$. We prove the assertion of Theorem 1 for the first equation. The proof for the second is similar. First we suppose that no $a_{i}$ equals 1 . Then we observe that $6 \mid(n+4)$ and we write $(2.9)$ as

$$
\begin{gathered}
n=2^{f_{0}} 5^{h_{0}} x_{0}^{3}, \quad n+1=3^{g_{1}} x_{1}^{3}, \quad n+2=2^{f_{2}} x_{2}^{3}, \\
n+4=2^{f_{4}} 3^{g_{4}} x_{4}^{3}, \quad n+5=5^{h_{5}} x_{5}^{3} .
\end{gathered}
$$

If $f_{2}=2$, then $f_{0}=f_{4}=1$ and (8.3) is not possible. Thus $f_{2}=1$ since no $a_{i}$ equals 1 . Suppose $f_{4}=2$. Then $a_{1} a_{4}=a_{2} a_{2} 3^{3}$, which contradicts Lemma 4. If $f_{4}=1$, then $f_{0}=1$ and hence $a_{0} a_{5}=a_{1} a_{4}\left(\frac{5}{3}\right)^{3}$, a contradiction to Lemma 4. If $f_{4}=0$, then $f_{0}=2$ and we have $a_{0} a_{5}=$ $a_{2} a_{2} 5^{3}$, a contradiction. Thus we may assume that one of $a_{1}, a_{2}, a_{4}$ is equal to 1 since $h_{0}>0$ and $h_{5}>0$. We have $a_{i} \in\{1,2,3,4,6,9,12,18,36\}$ for $i \in\{1,2,4\}$. Let $a_{1}=1$. Then $x_{1}>1$ and $a_{2} x_{2}^{3}-x_{1}^{3}=1$, which implies that $\left(x_{1}^{3}+1\right) /\left(x_{1}+1\right)=x_{2}^{\prime 3}$ or $3 x_{2}^{\prime 3}$ since $P\left(a_{2}\right) \leq 3$. Therefore $x_{1}=2,19$ by Nagell and Ljunggren (see Ribenboim [10, pp. 96, 105]). Hence $n+1=86859$, which is not possible since $5 \mid n$. The possibility 
$a_{2}=1$ is excluded similarly. Let $a_{4}=1$. Then $n+4 \equiv 0,1,8(\bmod 9)$. Thus $n=4,5,6(\bmod 9)$. Let $3 \mid a_{1}$. Then $a_{1}=3,6,12$ since $9 \nmid a_{1}$. If $a_{1}=6$ or 12 , then $a_{2}=1$, which is not possible. If $a_{1}=3$, then $a_{2}=$ 2 or 4 , which is not possible by Lemma 14 . Hence $3 \nmid a_{1}$. Then $a_{1}=2$ or 4 and $a_{2}=3$ since $9 \nmid(n+2)$. Finally, we apply Lemma 14 to exclude the above possibilities and our proof for the case $k=6$ is complete.

Let $k=5,7$. Then $a_{i}$ 's are composed of 2,3 if $k=5$, and of $2,3,5$ if $k=7$. Hence $f_{0}(k, 2) \geq 4$, which is not possible by Lemma 4 and Lemma 6 with $\ell^{\prime}=\ell-1, m_{1}=2, m_{2}=0$.

Let $k=4$. Then by Lemma 12 , we have either $n(n+2)(n+3)=y^{\ell}$ or $n(n+1)(n+3)=y^{\ell}$. We prove the assertion of Theorem 1 for the first equation. The details for the second equation are similar. By Lemma 4, we derive that no $a_{i}$ is equal to one. Then we conclude that $6 \mid n$. Then either

$$
n=2^{\ell-1} 3^{g_{0}} x_{0}^{\ell}, \quad n+2=2 x_{2}^{\ell}, \quad n+3=3^{g_{3}} x_{3}^{\ell}
$$

or

$$
n=2 \cdot 3^{g_{0}} x_{0}^{\ell}, \quad n+2=2^{\ell-1} x_{2}^{\ell}, \quad n+3=3^{g_{3}} x_{3}^{\ell} .
$$

We exclude the first possibility and the proof for the second is similar. We observe that $0<g_{0}<\ell, 0<g_{3}<\ell$ and $3^{g_{3}-1} x_{3}^{\ell}-2^{\ell-1} 3^{g_{0}-1} x_{0}^{\ell}=1$, which is not possible by Lemma 13 if $\ell \geq 5$. Let $\ell=3$. Then we apply Lemma 14 to $3^{g_{3}} x_{3}^{3}-2 x_{2}^{3}=1$ to get $g_{3}=2$. Then $g_{0}=1$ and $3 x_{3}^{3}-4 x_{0}^{3}=1$, which is not possible again by Lemma 14 .

Let $k=3$. It is clear that the product of two consecutive positive integers is never a power. Therefore, we need to consider only the equation $n(n+2)$ $=y^{\ell}$, which is not possible by Lemma 11 .

\section{References}

[1] M. Bennett, Rational approximation to algebraic numbers of small height: The diophantine equation $\left|a x^{n}-b y^{n}\right|=1$, J. Reine Angew. Math., to appear.

[2] H. Darmon and L. Merel, Winding quotients and some variants of Fermat's Last Theorem, ibid. 490 (1997), 81-100.

[3] P. Erdős, Note on the product of consecutive integers (II), J. London Math. Soc. 14 (1939), 245-249.

[4] - , On the product of consecutive integers III, Indag. Math. 17 (1955), 85-90.

[5] P. Erdős and J. L. Selfridge, The product of consecutive integers is never a power, Illinois J. Math. 19 (1975), 292-301.

[6] K. Györy, On the diophantine equation $n(n+1) \ldots(n+k+1)=b x^{l}$, Acta Arith. 83 (1998), 87-92.

[7] -, Power values of products of consecutive integers and binomial coefficients, in: Number Theory and Its Applications, S. Kanemitsu and K. Győry (eds.), Kluwer, 1999, 145-156. 
[8] M. Langevin, Plus grand facteur premier d'entiers en progression arithmétique, Sém. Delange-Pisot-Poitou, 18e année, 1976/77, no. 3, 6 pp.

[9] R. Marszałek, On the product of consecutive elements of an arithmetic progression, Monatsh. Math. 100 (1985), 215-222.

[10] P. Ribenboim, Catalan's Conjecture, Academic Press, 1994.

[11] K. A. Ribet, On the equation $a^{p}+2^{\alpha} b^{p}+c^{p}=0$, Acta Arith. 79 (1997), 7-16.

[12] O. Rigge, Über ein diophantisches Problem, 9th Congress Math. Scand. Helsingfors, 1938, Mercator, 1939, 155-160.

[13] B. Rosser and L. Schoenfeld, Approximate formulas for some functions of prime numbers, Illinois J. Math. 6 (1962), 64-94.

[14] J. W. Sander, Rational points on a class of superelliptic curves, J. London Math. Soc. 59 (1999), 422-434.

[15] N. Saradha, On perfect powers in products with terms from arithmetic progressions, Acta Arith. 82 (1997), 147-172.

[16] G. Shimura, Introduction to the Arithmetic Theory of Automorphic Functions, Princeton Univ. Press, 1971.

[17] T. N. Shorey, Perfect powers in products of integers from a block of consecutive integers, Acta Arith. 49 (1987), 71-79.

[18] - Some exponential diophantine equations, in: New Advances in Transcendence Theory, A. Baker (ed.), Cambridge Univ. Press, 1988, 352-365.

[19] - , Perfect powers in products of arithmetical progressions with fixed initial term, Indag. Math. 7 (1996), 521-525.

[20] - Exponential diophantine equations involving products of consecutive integers and related equations, in: Number Theory, R. P. Bambah, V. C. Dumir and R. J. HansGill (eds.), Hindustan Book Agency, 1999, 463-495.

[21] T. N. Shorey and Yu. V. Nesterenko, Perfect powers in products of integers from a block of consecutive integers (II), Acta Arith. 76 (1996), 191-198.

[22] T. N. Shorey and R. Tijdeman, Perfect powers in products of terms in an arithmetical progression, Compositio Math. 75 (1990), 307-344.

[23] - - - On the greatest prime factor of an arithmetical progression, in: A Tribute to Paul Erdős, A. Baker, B. Bollobás and A. Hajnal (eds.), Cambridge Univ. Press, 1990, 385-389.

[24] - - - Perfect powers in products of terms in an arithmetical progression III, Acta Arith. 61 (1992), 391-398.

[25] —, - Some methods of Erdös applied to finite arithmetic progressions, The Mathematics of Paul Erdős I, R. L. Graham and J. Nešetřil (eds.), Springer, 1997, 251-267.

[26] J. J. Sylvester, On arithmetical series, Messenger Math. 21 (1892), 1-19, 87-120.

School of Mathematics

Tata Institute of Fundamental Research

Homi Bhabha Road

Mumbai 400 005, India

E-mail: saradha@math.tifr.res.in shorey@math.tifr.res.in 